\title{
Equivalence Models for Quantified Boolean Formulas
}

\author{
Hans Kleine Büning ${ }^{1}$ and Xishun Zhao ${ }^{2}$ \\ 1 Department of Computer Science, \\ Universität Paderborn

\begin{abstract}
In this paper, the notion of equivalence models for quantified Boolean formulas with free variables is introduced. The computational complexity of the equivalence model checking problem is investigated in the general case and some restricted cases. We also establish a connection between the structure of some quantified Boolean formulas and the structure of models.
\end{abstract}

Keywords: quantified Boolean formula, equivalence model, model checking, complexity, equivalence, satisfiability

\section{Introduction}

The notion of models for formulas in $Q B F$ (i.e., the class of quantified Boolean formulas in prenex normal form without free variables) has been introduced in $[8,9]$. Generally speaking, an assignment for a formula in $Q B F$ is a mapping which maps each existential variable to a propositional formula over universal variables whose quantifiers precede the quantifier of the existential variable. An assignment $M$ is a model for a quantified formula $\Phi$ (with existential variables $\boldsymbol{y}=y_{1}, \cdots, y_{m}$ ) if the resulting formula $\Phi[\boldsymbol{y} / M]$ after replacing each existential variable by its corresponding formula (and removing existential quantifiers from the prefix) is true.

The notion of models for closed $Q B F$ can be easily extended to formulas in $Q B F^{*}$, the class of quantified Boolean formulas with (or without) free variables, by just allowing free variables occurring in the propositional formulas of assignments.

In this paper, we often write $\Phi=Q \phi(\boldsymbol{x}, \boldsymbol{y})$ and $\Phi(\boldsymbol{z})=Q \phi(\boldsymbol{x}, \boldsymbol{y}, \boldsymbol{z})$ for formulas in $Q B F$ and $Q B F^{*}$, respectively, with universal variables $\boldsymbol{x}=x_{1}, \cdots, x_{n}$, existential variables $\boldsymbol{y}=y_{1}, \cdots, y_{m}$, and free variables $\boldsymbol{z}=z_{1}, \cdots, z_{r}$.

Please note that if $M$ is a model for a formula $\Phi \in Q B F$ then $\Phi$ and $\Phi[\boldsymbol{y} / M]$ are equivalent. However, this is generally invalid for formulas in $Q B F^{*}$ and their models. For example, the formula $\Phi\left(z_{1}, z_{2}\right)=\exists y\left(z_{1} \vee y\right) \wedge\left(\neg y \vee z_{2}\right)$ is equivalent to the formula $\left(z_{1} \vee z_{2}\right)$. For $f_{y}\left(z_{1}, z_{2}\right)=1, M=\left(f_{y}\right)$ is a model, since $\Phi\left[y / f_{y}\right]=\left(z_{1} \vee 1\right) \wedge(0 \vee z) \approx z$ is satisfiable. But the resulting formula is not equivalent to the input formula $\Phi\left(z_{1}, z_{2}\right)$.

This motivates us to introduce and investigate equivalence models for formulas in $Q B F^{*}$, which deserve attention because quantified Boolean formulas can be used to represent Boolean functions with essentially small size. There are Boolean functions which can be represented by a formula in $Q B F^{*}$ with quadratic size while every propositional formula representing the same function has super-polynomial size [7]. An assignment $M$ is an equivalence model for a formula $\Phi \in Q B F^{*}$ if $\Phi$ and $\Phi[\boldsymbol{y} / M]$ are equivalent. We are interested in the equivalence model checking problem of determining whether an assignment is an equivalence model of a formula $\Phi \in Q B F^{*}$, and the equivalent model problem of deciding whether a formula in $Q B F^{*}$ has an equivalence model. Since the equivalence model checking problem involves testing the equivalence of two quantified formulas, the problem is $P S P A C E-$ complete. Without any restriction we will see that any $Q B F^{*}$ formula has an equivalence model. In this paper, we restrict the two problems to some subclasses of $Q B F^{*}$ and some models consisting of propositional formulas with special structures. 
We are also interested in discovering some connections between the structure of formulas in $Q B F^{*}$ and that of models. We will show that $Q 2-C N F^{*}$ formulas always have an equivalence model consisting of formulas in 1- $C N F \cup 1-D N F \cup\{0,1\}$.

In the remaining of this section we will recall and introduce some notations and terminologies.

The classes of proposition formulas such as $C N F, D N F, k-C N F, H O R N$ and so on, are defined as usual.

$Q B F$ is the class of closed quantified Boolean formula (i.e., without free variables). The formula $\Phi$ is in prenex normal form, if $\Phi=Q_{1} v_{1} \cdots Q_{n} v_{n} \phi$, where $Q_{i} \in\{\forall, \exists\}$ and $\phi$ is a propositional formula over variables $v_{1}, \cdots, v_{n} . Q_{1} v_{1} \cdots Q_{n} v_{n}$ is called the prefix and $\phi$ the matrix or kernel of $\Phi$. Usually, we simply write $\Phi=Q \phi$. A literal $x$ or $\neg x$ is called a universal resp. existential literal, if the variable $x$ is bounded by a universal quantifier resp. by an existential quantifier. A closed formula $\Phi \in Q B F$ with prenex form is called satisfiable or true, if there exists an assignment of truth values to the existential variables depending on the preceding universal variables, for which the propositional kernel of the formula is true. $Q C N F$ denotes the class of $Q B F$ formulas in prenex normal form with matrix in $C N F$, likewise for $Q k-C N F, Q H O R N$.

The classes $Q C N F^{*}, Q 2-C N F^{*}, Q H O R N^{*}$ are defined in the same way as $Q C N F, Q 2-C N F$, $Q H O R N$, respectively, except allowing free variables. A formula in $Q C N F^{*}$ is satisfiable if and only if there is a truth assignment for the free variables, such that for the truth assignment the closed $Q B F$ is true. The class $\exists^{*} C N F^{*}$ is a subset of $Q C N F^{*}$ in which any formula has a purely existential prefix and a $C N F$ kernel.

In our investigations we will make use of substitutions of existential variables by propositional formulas. For a quantified Boolean formula $\Phi$ with or without free variables $\Phi\left[y_{1} / f_{1}, \cdots, y_{m} / f_{m}\right]$ denotes the formula obtained by simultaneously substituting the occurrences of each variables $y_{i}$ by the formula $f_{i}$ and removing quantifiers of $y_{i}$. For $\Phi\left[y_{1} / f_{1}, \cdots, y_{m} / f_{m}\right], \boldsymbol{y}=y_{1}, \cdots, y_{m}$, and $M=\left(f_{1}, \cdots, f_{m}\right)$ we write $\Phi[\boldsymbol{y} / M]$.

\section{Models}

In this section we present two definitions of models for quantified Boolean formulas and prove some basic results. The first definition is based on satisfiability and has been investigated in $[8,9]$ for closed formulas.

Definition 1. (Satisfiability Model) [8]

Let $\Phi(\boldsymbol{z})=Q \phi(\boldsymbol{x}, \boldsymbol{y}, \boldsymbol{z})$ be a formula in $Q C N F^{*}$, where $\boldsymbol{x}=x_{1}, \cdots, x_{n}$ are universal variables, $\boldsymbol{y}=y_{1}, \cdots, y_{m}$ existential variables, and $\boldsymbol{z}=z_{1}, \cdots, z_{r}$ free variables. For propositional formulas $f_{y_{i}}$ over $\boldsymbol{z}$ and universal variables whose quantifiers precede $\exists y_{i}$, we say $M=\left(f_{y_{1}}, \cdots, f_{y_{m}}\right)$ is a (satisfiability) model for $\Phi(\boldsymbol{z})$ if and only if $\forall x_{1} \cdots \forall x_{n} \phi(\boldsymbol{x}, \boldsymbol{y}, \boldsymbol{z})[\boldsymbol{y} / M]$ is satisfiable.

If the propositional formulas $f_{y_{i}}$ belong to a class $K$, then $M$ is called a $K$-model for $\Phi(\boldsymbol{z})$.

For example, the formula $\Phi(z)=\forall x \exists y(x \vee y) \wedge(\neg x \vee \neg y) \wedge z$ is satisfiable and for $f_{y}(x, z)=\neg x$, $M=\left(f_{y}\right)$ is a model for $\Phi(z)$, because

$$
\forall x((x \vee y) \wedge(\neg x \vee \neg y) \wedge z)\left[y / f_{y}\right]=\forall x(x \vee \neg x) \wedge(\neg x \vee x) \wedge z
$$

is satisfiable (set $z=1)$.

The formula $\Phi\left(z_{1}, z_{2}\right)=\exists y\left(z_{1} \vee y\right) \wedge\left(\neg y \vee z_{2}\right)$ is logically equivalent to the formula $\left(z_{1} \vee z_{2}\right)$. For $f_{y}\left(z_{1}, z_{2}\right)=1, M=\left(f_{y}\right)$ is a model, since $\Phi\left(z_{1}, z_{2}\right)\left[y / f_{y}\right]=\left(z_{1} \vee 1\right) \wedge\left(0 \vee z_{2}\right) \approx z_{2}$ is satisfiable. But the formula $\Phi\left(z_{1}, z_{2}\right)\left[y / f_{y}\right]$ is not equivalent to the input formula $\Phi\left(z_{1}, z_{2}\right)$. For $g_{y}\left(z_{1}, z_{2}\right)=z_{2}$, however, we get the equivalence $\Phi\left(z_{1}, z_{2}\right)\left[y / g_{y}\right]=\left(z_{1} \vee z_{2}\right) \wedge\left(\neg z_{2} \vee z_{2}\right) \approx\left(z_{1} \vee z_{2}\right) \approx \Phi\left(z_{1}, z_{2}\right)$. That means, the substitution of the existential variable $y$ by the associated propositional formula $g_{y}$ preserves the image of the formula. This simple observation motivates a second definition of models for quantified Boolean formulas. 
Definition 2. (Equivalence Model)

Let $\Phi(\boldsymbol{z})=Q \phi(\boldsymbol{x}, \boldsymbol{y}, \boldsymbol{z})$ be a formula in $Q C N F^{*}$, where $\boldsymbol{x}=x_{1}, \cdots, x_{n}$ are universal variables, $\boldsymbol{y}=y_{1}, \cdots, y_{m}$ existential variables, and $\boldsymbol{z}=z_{1}, \cdots, z_{r}$ free variables. For propositional formulas $f_{y_{i}}$ over $\boldsymbol{z}$ and universal variables whose quantifiers precede $\exists y_{i}$, we say $M=\left(f_{y_{1}}, \cdots, f_{y_{m}}\right)$ is an equivalence model for $\Phi(\boldsymbol{z})$ if and only if $\Phi(\boldsymbol{z}) \approx \forall x_{1} \cdots \forall x_{n} \phi(\boldsymbol{x}, \boldsymbol{y}, \boldsymbol{z})[\boldsymbol{y} / M]$.

If the propositional formulas $f_{y_{i}}$ belong to a class $K$, then $M$ is called a $K$-equivalence model for $\Phi(\boldsymbol{z})$.

The formula $\Phi(z)=\forall x_{1} \forall x_{2} \exists y\left(x_{1} \vee y\right) \wedge\left(x_{2} \vee \neg y \vee z\right)$ is equivalent to $z$. For $f_{y}\left(x_{1}, x_{2}, z\right)=\neg x_{1}$, $M=\left(f_{y}\right)$ is an equivalence model, since $\Phi(z)\left[y / f_{y}\right]=\forall x_{1} \forall x_{2}\left(x_{1} \vee \neg x_{1}\right) \wedge\left(x_{2} \vee x_{1} \vee z\right) \approx \forall x_{1} \forall x_{2}\left(x_{2} \vee\right.$ $\left.x_{1} \vee z\right) \approx z$.

Obviously, any unsatisfiable formula has an equivalence model. In that case any propositional formula over the corresponding variables is an equivalence model.

Lemma 1. Any formula in $Q C N F^{*}$ has an equivalence model.

Proof. Suppose, we have a formula $\Phi(\boldsymbol{z})=Q \phi(\boldsymbol{z}, \boldsymbol{x}, \boldsymbol{y}) \in Q C N F^{*}$ equivalent to the Boolean function $F(\boldsymbol{z})$ with free variables $\boldsymbol{z}=z_{1}, \cdots, z_{m}$, universal variables $\boldsymbol{x}=x_{1}, \cdots, x_{t}$, and existential variables $\boldsymbol{y}=y_{1}, \cdots, y_{n}$. For fixed tuples of truth values $\boldsymbol{a} \in\{0,1\}^{m}$ the formula $\Phi(\boldsymbol{a})$ is a closed formula.

If the formula is true, then there is a satisfiabilty model $M^{a}=\left(f_{y_{1}}^{a}, \cdots, f_{y_{n}}^{a}\right)$. That means $\Phi(\boldsymbol{a})\left[\boldsymbol{y} / M^{\boldsymbol{a}}\right]$ is true.

If the formula is false, then for $M^{\boldsymbol{a}}=(0, \cdots, 0)$ the formula $\Phi(\boldsymbol{a})\left[\boldsymbol{y} / M^{\boldsymbol{a}}\right]$ is false.

Now, we combine these $2^{m}$ cases to an equivalence model as follows:

Let $\boldsymbol{x}^{i}=x_{1}, \cdots, x_{r_{i}}$ be the preceding universal variables for $y_{i}$. We define a Boolean function $f_{y_{i}}\left(\boldsymbol{z}, \boldsymbol{x}^{\boldsymbol{i}}\right)=f_{y_{i}}^{\boldsymbol{a}}\left(\boldsymbol{x}^{\boldsymbol{i}}\right)$, if $\boldsymbol{z}=\boldsymbol{a}$. Since for any Boolean function there is an equivalent propositional formula, for $M=\left(f_{y_{1}}, \cdots, f_{y_{n}}\right)$ we have $\Phi(\boldsymbol{z})[\boldsymbol{y} / M] \approx F(\boldsymbol{z}) \approx \Phi(\boldsymbol{z})$. Hence, $M$ is an equivalence model for $\Phi(\boldsymbol{z})$.

The next proposition states some simple observations for which we omit the proof.

Proposition 1. Let $\Phi(\boldsymbol{z})=Q \phi(\boldsymbol{x}, \boldsymbol{y}, \boldsymbol{z})$ be an arbitrary formula in $Q C N F^{*}, M=\left(f_{y_{1}}, \cdots, f_{y_{m}}\right)$ any sequence of propositional formulas.

1. $\Phi(\boldsymbol{z})[\boldsymbol{y} / M] \models \Phi(\boldsymbol{z})$. Moreover, $M$ is an equivalence model for $\Phi(\boldsymbol{z})$ if and only if $\Phi(\boldsymbol{z}) \models$ $\Phi(\boldsymbol{z})[\boldsymbol{y} / M]$

2. If $\Phi$ is closed (i.e., $\boldsymbol{z}$ is empty) and true, then $M$ is a (satisfiability) model for $\Phi$ if and only if $M$ is an equivalence model for $\Phi$.

Let $K$ be a class of propositional formulas and $X \subseteq Q C N F^{*}$. We are mainly interested in the following problems:

$K$-Equivalence Model Checking Problem for $X$ :

Instance: A formula $\Phi \in X$ and $M=\left(f_{1}, \cdots, f_{n}\right)$ a sequence of propositional formulas $f_{i} \in K$.

Query: Is $M$ a $K$-equivalence model for $\Phi$ ?

\section{$K$-Equivalence Model Problem for $X$ :}

Instance: A formula $\Phi \in X$.

Query: Does there exist a $K$-equivalence model $M$ for $\Phi$ ?

A procedure for deciding whether $M$ is an equivalence model for $\Phi(\boldsymbol{z})$ is as follows:

1. Substitute the existential variables by the associated propositional formulas and remove from the prefix the existential quantifiers.

2. Test whether the resulting formula is equivalent to the input formula.

Because the equivalence problem between two quantified formulas is PSPACE-complete even if one of them is very simple, we obtain the following lemma.

Lemma 2. The equivalence model checking problem for $Q C N F^{*}$ is PSPACE-complete. 
Proof. Obviously, the equivalence model checking problem is in PSPACE, since the satisfiability and the equivalence problem for quantified Boolean formulas are in PSPACE. We prove the $P S P A C E$-hardness from a reduction of the PSPACE-complete evaluation problem for $Q C N F$ [7]. We associate to a closed formula $\Phi=Q \phi \in Q C N F$ for a new variable $y$ the formula $\Phi^{\prime}=\exists y Q(\phi \wedge y)$. Then, $\Phi$ is true if and only if $\Phi^{\prime}$ is true. Suppose, $\Phi$ has the existential variables $\boldsymbol{y}=y_{1}, \cdots, y_{n}$. For $M=\left(f_{y}, f_{y_{1}}, \cdots f_{y_{n}}\right)$, where all Boolean functions are the constant 0 , that means $f_{y}=0, f_{y_{i}}=0$, $\Phi^{\prime}[\boldsymbol{y} / M]$ is false. Hence, $M$ is an equivalence model for $\Phi^{\prime}$ if and only if $\Phi$ is false.

That means, equivalence model checking is much harder than satisfiability model checking which has been shown in [8] to be $c o N P$-complete for $Q C N F$.

The next theorem states that the upper bound for the complexity of the equivalence checking problem for classes $X$ depends on the complexity of the satisfiability problem for $X$. We say a class $X \subseteq Q C N F^{*}$ is closed under constant-substitutions if and only if for every formula $\Phi(\boldsymbol{z}) \in X$ and for all combinations of constants $\boldsymbol{a}$ the formula $\Phi(\boldsymbol{z})[\boldsymbol{z} / \boldsymbol{a}]=\Phi(\boldsymbol{a})$ is in $X$.

We recall the notion of the polynomial-time hierarchy. $\Sigma_{2}^{P}$ is a class of problems defined as $(k \geq 0): \Sigma_{0}^{P}=\Pi_{0}^{P}=P$ the class of polynomial-time solvable problems, $\Sigma_{k+1}^{P}=N P^{\Sigma_{k}^{P}}, \Pi_{k+1}^{P}=$ $c o-\Sigma_{k+1}^{P}$. Thus, $N P=\Sigma_{1}^{P}$ and $c o N P=\Pi_{1}^{P}$. Relationships between prefix classes of $Q B F^{*}$ and classes of the polynomial-time hierarchy has been shown for example in [15].

Theorem 1. For every class $X \subseteq Q C N F^{*}$ which is closed under constant-substitutions we have

1. If $X \cap Q S A T$ is polynomial-time solvable (i.e., in $\Sigma_{0}^{P}=\Pi_{0}^{P}$ ), then the equivalence model checking problem for $X$ is in coNP $=\Pi_{1}^{P}$.

2. For $k \geq 1$, if $X \cap Q S A T$ is in $\Sigma_{k}^{P}$, then the equivalence model checking problem for $X$ is in $\Pi_{k}^{P}$.

3. For $k \geq 1$, if $X \cap Q S A T$ is in $\Pi_{k}^{P}$, then the equivalence model checking problem for $X$ is in $\Pi_{k+1}^{P}$.

Here, QSAT is the class of all satisfiable formulas in $Q C N F^{*}$.

Proof. For any $\Phi(\boldsymbol{z})=Q \phi(\boldsymbol{x}, \boldsymbol{y}, \boldsymbol{z})$ and any sequence $M=\left(f_{y_{1}}, \cdots, f_{y_{m}}\right)$ of propositional formulas. we have the following equivalence relations.

$$
\begin{aligned}
& M \text { is not an equivalence model for } \Phi(\boldsymbol{z}) \\
& \Leftrightarrow \Phi(\boldsymbol{z}) \not \varpi \Phi(\boldsymbol{z})[\boldsymbol{y} / M] \Leftrightarrow \Phi(\boldsymbol{z}) \not \models \Phi(\boldsymbol{z})[\boldsymbol{y} / M] \\
& \Leftrightarrow \exists \boldsymbol{z}:(\Phi(\boldsymbol{z}) \text { is true and } \Phi(\boldsymbol{z})[\boldsymbol{y} / M] \text { is false }) \\
& \Leftrightarrow \exists \boldsymbol{z}:\left(\Phi(\boldsymbol{z}) \text { is true and } \forall x_{1} \cdots \forall x_{n} \phi(\boldsymbol{x}, \boldsymbol{z}, \boldsymbol{y})[\boldsymbol{y} / M] \text { is false }\right) \\
& \Leftrightarrow \exists \boldsymbol{z}:\left(\Phi(\boldsymbol{z}) \text { is true and } \exists x_{1} \cdots \exists x_{n} \neg \phi(\boldsymbol{x}, \boldsymbol{y}, \boldsymbol{z})[\boldsymbol{y} / M] \text { is true }\right) \\
& \Leftrightarrow \exists \boldsymbol{z} \exists \boldsymbol{x}^{\prime}:\left(\Phi(\boldsymbol{z}) \text { is true and } \neg \phi\left(\boldsymbol{x}^{\prime}, \boldsymbol{y}, \boldsymbol{z}\right)\left[\boldsymbol{y} / M^{\prime}\right] \text { is true }\right) \text {. }
\end{aligned}
$$

The propositional formula $\neg \phi\left(\boldsymbol{x}^{\prime}, \boldsymbol{y}, \boldsymbol{z}\right)\left[\boldsymbol{y} / M^{\prime}\right]$ contains the variables $\boldsymbol{x}^{\prime}$ and $\boldsymbol{z}$, where $\boldsymbol{x}^{\prime}:=x_{1}^{\prime}, \cdots, x_{n}^{\prime}$ and $M^{\prime}$ is the result of renaming $x_{i}$ by $x_{i}^{\prime}$ for every $i$. Whether for fixed values for $\boldsymbol{x}^{\prime}$ and $\boldsymbol{z}$ the formula evaluates to true, can be decided in linear time. Please note that for fixed values for $\boldsymbol{z}$ the formula $\Phi(\boldsymbol{z})$ is in $X$.

If the satisfiability of formulas in $X$ is solvable in polynomial time, then the non-equivalence model checking problem is in $N P=\Sigma_{1}^{P}$. Hence, the complementary problem - the equivalence model checking problem - is in $\operatorname{coNP}=\Pi_{1}^{P}$.

If the satisfiability problem for formulas in $X$ is in $\Sigma_{k}^{P}$ then the problem whether $\exists \boldsymbol{x} \Phi(\boldsymbol{x})$ is satisfiable remains in $\Sigma_{k}^{P}$. Hence, the equivalence model checking problem is in $\Pi_{k}^{P}$.

If the satisfiability problem for formulas in $X$ is in $\Pi_{k}^{P}$ then the problem whether $\exists \boldsymbol{x} \Phi(\boldsymbol{x})$ is satisfiable is in $\Sigma_{k+1}^{P}$. Hence, the equivalence model checking problem is in $\Pi_{k+1}^{P}$.

Please note, that Theorem 1 establishes only an upper bound for the complexities. Classes with a tractable satisfiability problem are $Q_{2} 2-C N F^{*}$ and $Q H O R N^{*}[1,4]$, whereas the satisfiability problem for $\exists * C N F^{*}$ is obviuosly $N P$-complete. With respect to the completeness of the various problems we can prove

Lemma 3. The equivalence model checking problems for $\exists^{*} C N F^{*}, Q 2-C N F^{*}$ and $Q H O R N^{*}$ are coNP-complete. 
Proof. The $\operatorname{coNP}$-hardness of the eqivalence checking problem for $\exists^{*} C N F^{*}$ follows from a reduction from the $c o N P$-complete unsatisfiability problem for $C N F$. We associate to every propositional $C N F$ formula $\phi(\boldsymbol{x})$ the formula $\Phi(\boldsymbol{x})=\exists y(y \wedge \phi(\boldsymbol{x}))$ and $M=\left(f_{y}(\boldsymbol{x})\right)$ with $f_{y}(\boldsymbol{x})=0$. The formula $\Phi(\boldsymbol{x})$ is equivalent to $\phi(\boldsymbol{x})$. Substituting the existential variable $y$ by the model function $f_{y}$ we obtain $\Phi(\boldsymbol{x})\left[y / f_{y}(\boldsymbol{x})\right]=0 \wedge \phi(\boldsymbol{x}) \approx 0$. Hence, $M$ is an equivalence model for $\Phi(\boldsymbol{x})$ if and only if $\phi(\boldsymbol{x})$ is unsatisfiable.

That the problem is in $c o N P=\Pi_{1}^{P}$ follows from Theorem 1, because $\exists^{*} C N F$ is closed under constant-substitutions and the satisfiability problem is in $N P$.

The other classes Q2-CNF* and $Q H O R N^{*}$ are closed under constant-substitution and their satisfiability problems are solvable in polynomial time [7]. Hence, by Theorem 1 the problems are in $\operatorname{coNP}$. The coNP-hardness follows from a reduction from the coNP-complete tautology problem for $D N F$. We associate to a formula $\psi \in D N F$ over the variables $x_{1}, \cdots, x_{n}$ the quantified Boolean formula $\Phi=\forall x_{1} \cdots \forall x_{n} \exists y: \neg y$ and $M=\left(f_{y}\left(x_{1}, \cdots, x_{n}\right)\right)$, where $f_{y}\left(x_{1}, \cdots, x_{n}\right)=\neg \psi$. Then, $M$ is an equivalence model for $\Phi$ if and only if $\neg f_{y}\left(x_{1}, \cdots, x_{n}\right)=\psi \in D N F$ is a tautology.

\section{Special Classes of Models}

In this section we investigate the problems for various classes of model formulas and input formulas. Some of the results are depicted in figure 1 . Two classes $K_{0}$, the set of constants 0 and 1 , and $K_{2}$, the set of monomials, are defined as $K_{0}:=\{f \mid f$ is 0 or 1$\}$ and $K_{2}:=\{f \mid \exists I \subseteq\{1, \cdots, n\}$ : $\left.f\left(x_{1}, \ldots, x_{n}\right)=\bigwedge_{i \in I} x_{i}, n \in \mathbf{N}\right\} \cup K_{0}$

\begin{tabular}{|l|l|l|}
\hline \hline Boolean functions & $Q C N F^{*}$-class & equivalence model checking \\
\hline$K_{0}=\{0,1\}$ & $Q C N F$ & $P S P A C E$-complete \\
\hline $1-C N F \cup 1-D N F \cup\{0,1\}$ & $Q 2-C N F^{*}$ & polytime \\
\hline $1-D N F$ & $Q H O R N^{*}$ & coNP-complete \\
\hline$K_{2}$ & $Q H O R N^{*}$ & polytime \\
\hline \hline
\end{tabular}

Figure 1

For a formula $\Phi=\exists^{*} \phi \in \exists^{*} C N F^{*}$, if the kernel $\phi$ is satisfiable then $\Phi$ has a $K_{0}$-model. However, this is not true for equivalence models. The formula $\Phi\left(z_{1}, z_{2}\right)=\exists y\left(z_{1} \vee y\right) \wedge\left(\neg y \vee z_{2}\right)\left(\approx\left(z_{1} \vee z_{2}\right)\right)$ has no $K_{0}$-equivalence model, since

$$
\begin{aligned}
& \Phi\left(z_{1}, z_{2}\right)[y / 0]=\left(z_{1} \vee 0\right) \wedge\left(1 \vee z_{2}\right) \approx\left(z_{1}\right) \not\left(z_{1} \vee z_{2}\right) \\
& \Phi\left(z_{1}, z_{2}\right)[y / 1]=\left(z_{1} \vee 1\right) \wedge\left(0 \vee z_{2}\right) \approx\left(z_{2}\right) \not \approx\left(z_{1} \vee z_{2}\right)
\end{aligned}
$$

Since the equivalence problem for $Q C N F$ formulas is PSPACE-complete even if one of the formulas is very simple, we have the following lemma.

\section{Lemma 4.}

1. The $K_{0}$-equivalence model checking problem for QCNF is PSPACE-complete.

2. The $K_{0}$-equivalence model problem for QCNF is PSPACE-complete.

Proof. Ad 1: (see proof of Lemma 2)

$A d$ 2: Obviously, the problem is in PSPACE. For the PSPACE-hardness, we associate to $Q C N F$ formulas $\Phi=Q \phi(\boldsymbol{x}, \boldsymbol{y})$ for new variables $x_{0}$ and $y_{0}$ the $Q C N F$ formula $\Phi^{\prime}:=\forall x_{0} \exists y_{0} Q(\phi(\boldsymbol{x}, \boldsymbol{y}) \wedge$ $\left.\left(x_{0} \vee y_{0}\right) \wedge\left(\neg x_{0} \vee \neg y_{0}\right)\right) . \Phi$ is true if and only if $\Phi^{\prime}$ is true, since $\Psi:=\forall x_{0} \exists y_{0}\left(\left(x_{0} \vee y_{0}\right) \wedge\left(\neg x_{0} \vee \neg y_{0}\right)\right)$ is true for example with $f_{y_{0}}\left(x_{0}\right)=\neg x_{0}$. But $\Psi$ has no $K_{0}$-equivalence model. That can be seen by a case distinction $y_{0}=0$ and $y_{0}=1$. Therefore, if $\Phi$ is true, then $\Phi^{\prime}$ has no $K_{0}$-equivalence model. Suppose $\Phi$ is false, then $\Phi^{\prime}$ is false. Thus, $\Phi^{\prime}$ has a $K_{0}$-equivalence model. Altogether, $\Phi$ is false if and only if $\Phi^{\prime}$ has a $K_{0}$-equivalence model. Since the evaluation problem for $Q C N F$ is PSPACE-complete, we have shown our desired result.

Satisfiable Q2-CNF formulas have always a satisfiability model consisting of formulas of the form $f_{y}(\boldsymbol{x})=(\neg) x_{i}$ for some $i, f_{y}(\boldsymbol{x})=0$, or $f_{y}(\boldsymbol{x})=1$. For $Q 2-C N F^{*}$ these model formulas are not sufficient as the following example shows $\Phi\left(z_{1}, z_{2}\right)=\exists y\left(z_{1} \vee y\right) \wedge\left(z_{2} \vee y\right) \wedge\left(\neg y \vee z_{3}\right) \wedge\left(\neg y \vee z_{4}\right)$. The proof is straight forward by a case distinction. We will see that the class of models defined as $\mathcal{B}=1-C N F \cup 1-D N F \cup\{0,1\}$ characterizes in a certain sense equivalence models for $Q^{2}-C N F^{*}$. 


\section{Theorem 2.}

1. Any formula in $Q 2-C N F^{*}$ has a $\mathcal{B}$-equivalence model.

2. The $\mathcal{B}$-equivalence model checking for $Q^{2}-C N F^{*}$ is solvable in polynomial time.

Proof. Ad 1: Idea of the proof. Suppose, we have a formula $\Phi(\boldsymbol{z})=Q \phi(\boldsymbol{x}, \boldsymbol{y}, \boldsymbol{z}) \in Q 2-C N F^{*}$. If $\Phi(\boldsymbol{z})$ is unsatisfiable, then there is a $\{0,1\}$-equivalence model, and therefore a $\mathcal{B}$-equivalence model. Now, we assume the satisfiability of the input formula. In a first step we apply the Q-resolution as long as possible with $\Phi(\boldsymbol{z})$ [7]. The resulting formula, called $\Psi(\boldsymbol{z})$, is again in $Q^{2}-C N F^{*}$ and for any truth assignment for $\boldsymbol{z}, \Phi(\boldsymbol{z})$ is true if and only if $\Psi(\boldsymbol{z})$ is true. Next we will define $f_{y_{j}}$ for each $y_{j}$ by means of the derived unit clauses.

case 1. $y_{j}$ or $\neg y_{j}$ occurs in $\Psi(\boldsymbol{z})$ as a unit clause. Then define $f_{y_{j}}=1$ or $f_{y_{j}}=0$ accordingly.

case 2. $y_{j}$ occurs in a $\exists$-unit clause (i.e., a clause with one existential literal and the other literal is universal), but $\neg y_{i}$ does not occur in any $\exists$-unit clause. Let $w_{1} \vee y_{j}, \cdots, w_{k} \vee y_{j}$ be all the $\exists$-unit clause containing $y_{j}$. Then define $f_{y_{j}}=\neg w_{1} \vee \cdots \vee \neg w_{k}$.

case 3. $\neg y_{j}$ occurs in a $\exists$-unit clause, but $y_{i}$ does not occur in any $\exists$-unit clause. Let $w_{1} \vee$ $\neg y_{j}, \cdots, w_{k} \vee \neg y_{j}$ be all the $\exists$-unit clause containing $\neg y_{j}$. Then define $f_{y_{j}}=w_{1} \wedge \cdots \wedge w_{k}$.

case 4. Both $y_{j}$ and $\neg y_{j}$ occur in some $\exists$-unit clauses. Since $\Psi$ is satisfiable, there are exactly two clauses containing $y_{j}$ or $\neg y_{j}$, and they must be of the form $w \vee y_{j}$ and $\neg w \vee \neg y_{j}$. Then define $f_{y_{j}}=\neg w$.

case 5. $y_{j}$ or $\neg y_{j}$ is derivable from free-unit clauses (by a free-unit clause we mean a clause with at most one existential literal and the other literals are literals over free variables). Then define $f_{y_{j}}=1$ or $f_{y_{j}}=0$ accordingly.

case 6. $y_{j}$ or $\neg y_{j}$ is a pure literal. Then define $f_{y_{j}}=1$ or $f_{y_{j}}=0$ accordingly.

case 7. Note cases 1-6. Let $y_{j} \vee v_{1}, \cdots, y_{j} \vee v_{k}$ and $\neg y_{j} \vee u_{1}, \cdot, \neg y_{j} \vee u_{r}$. Then define $f_{y_{j}}$ either to be $\neg v_{1} \vee \cdots \vee \neg v_{k}$ or to be $u_{1} \wedge \cdots \wedge u_{r}$.

case 8. There are no free-unit clauses containing $y_{j}$ or $\neg y_{j}$. That is, $y_{j}$ has nothing to do with free variables and existential variables. Thus, in this case $f_{y_{j}}$ is either 0 or 1 .

It is not hard to see that in any case $\Psi(\boldsymbol{z})\left[y_{j} / f_{y_{j}}\right]$ is true if and only if $\Psi(\boldsymbol{z})$ is true for any truth assignment for $\boldsymbol{z}$. Consequently, $\left(f_{y_{1}}, \cdots, f_{y_{m}}\right)$ is an equivalence model for $\Phi(\boldsymbol{z})$.

$A d$ 2: Let $\Phi(\boldsymbol{z})=Q \phi(\boldsymbol{x}, \boldsymbol{y}, \boldsymbol{z})$ be in $Q 2-C N F^{*}$. For a sequence of propositional formulas $M=$ $\left(f_{y_{1}}, \cdots, f_{y_{m}}\right)$, where $f_{y_{i}} \in \mathcal{B}$, we want to decide whether $M$ is an equivalence model for $\Phi(\boldsymbol{z})$.

At first we can calculate by applying a polytime algorithm to $\Phi(\boldsymbol{z})$ a logically equivalent propositional formula $F(\boldsymbol{z}) \in{ }^{2}-C N F$. The length of $F(\boldsymbol{z})$ is bound by $O\left(|\phi|^{2}\right)$ (see Theorem 7.4.6 and Theorem 7.6.1 in[7]). In the next step we substitute in the initial formula the existential variables $y_{i}$ by the model-functions $f_{y_{i}}$. That means, we have $\Phi(\boldsymbol{z})[\boldsymbol{y} / M]=\forall x_{1} \cdots \forall x_{n} \phi(\boldsymbol{x}, \boldsymbol{y}, \boldsymbol{z})[\boldsymbol{y} / M]$. Please note that $\Phi(\boldsymbol{z})[\boldsymbol{y} / M]$ may not in $Q C N F^{*}$. However, it can be transformed in polynomial time into an equivalent formula with $C N F$ kernel by applying the distributivity law. The result is denoted as $\Psi(\boldsymbol{z})$ (which still contains only universal quantifiers). Further, we can calculate in polynomial time an equivalent propositional formula $G(\boldsymbol{z})$ of length less or equal than the length of $\Psi(\boldsymbol{z})$. If $\Psi(\boldsymbol{z})$ contains a $\forall$-clause then $G(z)$ is false. Otherwise, $G(z)$ is obtained by deleting all universal literals and removing the quantifiers. It is not difficult to see that $G(\boldsymbol{z})$ and $\Psi(\boldsymbol{z})$ are equivalent. Finally it remains to decide whether $F(\boldsymbol{z}) \models G(\boldsymbol{z})$. Since $F(\boldsymbol{z})$ is a propositional 2-CNF formula that can be done in polynomial time.

Altogether, we have an polytime procedure for the $\mathcal{B}$-equivalence model checking problem for 2-CNF*.

For $Q H O R N^{*}$ the regular equivalence problem - whether two quantified Horn formulas are equivalent - is $\operatorname{coNP}$-complete. Further, any $Q H O R N^{*}$ formula is equivalent to a $H O R N$ formula, but sometimes of length essentially different [7]. The next lemma shows that for very simple model formulas the $c o N P$-completeness persist.

Lemma 5. The 1-DNF-equivalence model checking problem for $Q H O R N^{*}$ is coNP-complete.

Proof. By Theorem 1 the problem is in coNP. We show the coNP-hardness by a reduction from the coNP-complete tautology problem for $3-D N F$ formulas. We associate to the $D N F$ formula $\psi=\bigvee_{1 \leq i \leq m}\left(L_{i, 1} \wedge L_{i, 2} \wedge L_{i, 3}\right)$ with literals $L_{i, j}$ over the variables $z_{1} \cdots, z_{r}$ the quantified Horn 
formula $\Phi(\boldsymbol{z})=\exists y \exists y_{1} \cdots \exists y_{m}\left(\neg y_{1} \vee \cdots \vee \neg y_{m}\right) \wedge\left(\neg z_{1} \vee \cdots \vee \neg z_{r} \vee \neg y\right)$ and $M=\left(f_{y}, f_{y_{1}}, \cdots, f_{y_{m}}\right)$, where $f_{y}(\boldsymbol{z})=\left(\neg z_{1} \vee \cdots \vee \neg z_{r}\right), f_{y_{i}}(\boldsymbol{z})=\left(\neg L_{i, 1} \vee \neg L_{i, 2} \vee \neg L_{i, 3}\right) \in 1$-DNF. $\Phi(\boldsymbol{z})$ is always true, that means equivalent to the constant 1 .

We have $\Phi(\boldsymbol{z})[\boldsymbol{y} / M]=\left(\neg f_{y_{1}}(\boldsymbol{z}) \vee \cdots \vee \neg f_{y_{m}}(\boldsymbol{z})\right) \wedge\left(\neg z_{1} \vee \cdots \vee \neg z_{r} \vee\left(z_{1} \wedge \cdots \wedge z_{n}\right)\right) \approx\left(\neg f_{y_{1}}(\boldsymbol{z}) \vee\right.$ $\left.\cdots \vee \neg f_{y_{m}}(\boldsymbol{z})\right)$ and this formula is equivalent to $\Phi(\boldsymbol{z})$ if and only if the propositional DNF formula $\psi=\left(\neg f_{y_{1}}(\boldsymbol{z}) \vee \cdots \vee \neg f_{y_{m}}(\boldsymbol{z})\right)$ is a tautology.

Hence, our 1-DNF-equivalence model checking problem is coNP-complete.

With respect to the satisfiability models, we know that every satisfiable $Q H O R N$ has a $K_{2}{ }^{-}$ model [8]. That does not hold for the equivalence model and $Q H O R N^{*}$. The formula

$$
\Phi_{n}\left(z_{1,1}, \cdots, z_{n, n}\right):=\exists y_{1} \cdots \exists y_{n}\left(\neg y_{1} \vee \cdots \vee \neg y_{n}\right) \wedge \bigwedge_{1 \leq i, j \leq n}\left(y_{i} \vee \neg z_{i, j}\right)
$$

is equivalent to $\bigvee_{1 \leq i \leq n}\left(\neg z_{i, 1} \wedge \cdots \wedge \neg z_{i, n}\right) . M=\left(f_{y_{1}}, \cdots, f_{y_{n}}\right)$ is an equivalence model for $\Phi_{n}$ if $f_{y_{i}}\left(z_{1,1}, \cdots, z_{n, n}\right) \stackrel{(}{=}\left(z_{i, 1} \vee \cdots \vee z_{i, n}\right)$. But $\Phi_{n}\left(z_{1,1}, \cdots, z_{n, n}\right)$ has no $K_{2}$-equiv-model.

Lemma 6. The $K_{2}$-equivalence model checking for $Q H O R N^{*}$ is solvable in polynomial time.

Proof. Suppose $\Phi(\boldsymbol{z})=Q \phi(\boldsymbol{x}, \boldsymbol{y}, \boldsymbol{z}) \in Q H O R N^{*}$ and $M=\left(f_{y_{1}}, \cdots, f_{y_{m}}\right)$, where $f_{y_{i}} \in K_{2}$. That means, if $\boldsymbol{x}^{i}=x_{1}, \cdots, x_{r_{i}}$ are the preceding universal variables for $y_{i}$, then we have $f_{y_{i}}\left(\boldsymbol{z}, \boldsymbol{x}^{\boldsymbol{i}}\right)=$ $\bigwedge_{j \in J_{i}} v_{j}, v_{j} \in\left\{x_{1}, \cdots, x_{n}, z_{1}, \cdots, z_{r}\right\}$ for some $J_{i}, f_{y_{i}}=0$, or $f_{y_{i}}=1$.

If the formula $\Phi(\boldsymbol{z}) \in Q H O R N^{*}$ is unsatisfiable, which can be decided in polynomial time, then $M$ is an equivalence model for the formula. We continue assuming the formula is satisfiable.

The substitution $\Phi(\boldsymbol{z})[\boldsymbol{y} / M]$ can lead to a non-Horn kernel. Since every clause in the kernel of the input formula $\Phi(\boldsymbol{z})$ contains at most one positive literal, by the distributivity law we can transform in polynomial time the formula $\Phi(\boldsymbol{z})[\boldsymbol{y} / M]$ into a universally quantified $Q H O R N^{*}$ formula, say $\Psi(\boldsymbol{z})=\forall \boldsymbol{x} \bigwedge \psi_{j}(\boldsymbol{x}, \boldsymbol{z})$. We can simplify the formula to obtain an equivalent propositional Horn formula $\Lambda \psi_{j}^{\prime}(\boldsymbol{z})$ by removing the universal variables and all quantifiers. To test whether $M$ is an equivalence model, it suffices to decide whether $\Phi(\boldsymbol{z}) \models \psi_{j}^{\prime}(\boldsymbol{z})$, that means $\Phi(\boldsymbol{z}) \wedge \neg \psi_{j}^{\prime}(\boldsymbol{z})$ is unsatisfiable. But that is the problem of deciding whether a $Q H O R N^{*}$ formula is satisfiable and this problem is solvable in polynomial time.

\section{Conclusion and Outlook}

The results presented in the paper are a first step in understanding the structure of equivalence models and the complexity of the problems. There are various open problems. Take $Q H O R N^{*}$ as an example, try to establish a class of propositional formulas $K \subseteq C N F$ with the following properties:

1. Any formula in $Q H O R N^{*}$ has a $K$-equivalence model.

2. An $K$-equivalence model for $\Phi(\boldsymbol{z}) \in Q H O R N^{*}$ can be constructed in polynomial time.

3. The $K$-equivalence model checking problem for $Q H O R N^{*}$ is solvable in polynomial time.

\section{References}

1. B. Aspvall, B., M. F. Plass, M. F., and Tarjan, R. E.: A Linear-Time Algorithm for Testing the Truth of Certain Quantified Boolean Formulas, Information Processing Letters, 8 (1979), 121-123

2. Cadoli, M., Schaerf, M., Giovanardi, A., and Giovanardi, M.: An Algorithm to Evaluate Quantified Boolean Formulas and its Evaluation, In: highlights of Satisfiability Research in the Year 2000, IOS Press, 2000.

3. Cook, S., Soltys, M.: Boolean Programs and Quantified Propositional Proof Systems, Bulletin of the Section of Logic, $\mathbf{2 8}$ (1999), 119-129.

4. Flögel, A., Karpinski, M., and Kleine Büning, H.: Resolution for Quantified Boolean Formulas, Information and Computation 117 (1995), 12-18

5. Garey, M.R., Johnson, D.S.: Computers and Intractability: A Guide to the Theory of NP-Completeness. W.H. Freeman Company, San Francisco, 1979.

6. Giunchiglia, E., Narizzano, M., and Tacchella, A.: QuBE: A System for Deciding Quantified Boolean Formulas, In: Proceedings of IJCAR, Siena, 2001. 
7. Kleine Büning, H., Lettmann, T.: Propositional Logic: Deduction and Algorithms, Cambridge University Press, 1999.

8. Kleine Büning, H., Subramani, K., and Zhao, X.: On Boolean Models for Quantified Boolean Horn Formulas, SAT 2003, Italy. Lecture Notes in Computer Science, 2919 93-104, 2004.

9. Kleine Büning, H., Zhao, X.: On Models for Quantified Boolean Formulas, to appear in LNCS, 2004.

10. Letz, R.: Advances in Decision Procedure for Quantified Boolean Formulas, In: Proceedings of IJCAR, Siena, 2001.

11. Meyer, A. R., Stockmeyer, L. J.: Word Problems Requiring Exponential Time, In: Preliminary Report, Proc. $5^{\text {th }}$ Ann. Symp. on Theory of Computing, (1973), pp 1-9

12. Papadimitriou, C. H.: Computational Complexity, Addison-Wesley, New York, 1994.

13. Rintanen, J.T.: Improvements to the Evaluation of Quantified Boolean Formulae, In: Proceedings of IJCAI, 1999.

14. Schaefer, T.J.: The Complexity of Satisfiability Problem, In: Proceedings of the 10th Annual ACM Symposium on Theory of Computing (ed. A. Aho), 216-226, New York City, ACM Press, 1978.

15. Stockmeyer, L. J.: The Polynomial-Time Hierarchy, In: Theoretical Computer Science, 3(1977), 1-22. 